\title{
Nachhaltige Stärkung des ländlichen Raumes
}

\section{Rahmenbedingungen}

Die hier präsentierten Überlegungen sind nahtlos eingebettet in die internationale Fachdiskussion um «Nachhaltige Regionalentwicklung». ${ }^{1}$ Dabei sollen unter dem Stichwort «Kohäsion» sowohl der Stadt-Land-Verbund wie auch die regionale Wirtschaftsstruktur und die Umweltvorsorge (als gemeinsame, zusammengehörende Aufgaben) gefördert und gestärkt werden. Auch die «Raumordnung Schweiz» basiert auf diesem Grundkonzept (BRP 1996; BRP 1998).

Nachhaltigkeit ${ }^{2}$ ist die Basis der Diskussion über zukunftsfähige Raumentwicklung; dabei handelt es sich nicht nur um ein Gebot der Vernunft und Verantwortung, sondern - verbindlicher - um einen ungeschriebenen Verfassungsgrundsatz. ${ }^{3}$ Seit der Ratifizierung der RioDeklaration besteht zudem eine entsprechende staatsvertragliche Verpflichtung, welche ihren Niederschlag im Rio-Nachfolgeprozeß finden muß, und zwar konkret in allen relevanten Politikfeldern, also auch in der Raumordnungspolitik.

Aus wirtschaftsgeographischer Sicht sind insbesondere auch einige Hinweise auf ökonomische Aspekte der Raumentwicklung nötig; sie sind in der bisherigen Debatte wohl eher zu wenig beachtet worden. Zu erwähnen sind hier insbesondere Investitionsstrategien und ihre Folgekosten, das Problem der Kostenwahrheit sowie die Finanzierbarkeit unserer Mobilitätssysteme, welche erst aus den statischen Raumstrukturen funktionsfähige dynamische Systeme machen. ${ }^{4}$

Im Zeitalter der "Zwischenstadt» (als Hybridform zwischen Stadt und Land) wird die Abgrenzung des «Ländlichen Raumes» kontrovers (SIEVERTS 1997). Der Terminologie in den «Grundzügen der Raumordnung Schweiz» folgend (BRP, 1996/23), fallen darunter nicht nur landwirtschaftliche Gebiete außerhalb der Siedlungen, sondern alle nichtstädtischen Gebiete, also auch Dörfer und Kleinzentren mit ihren Bauzonen, Gewerbeund Dienstleistungsbetrieben, mit ihren urban orientierten Wegpendlern. Diese Differenzierung ist entscheidend für die Beantwortung der Frage: Soll der ländliche Raum gestärkt werden durch die Öffnung der Landwirtschaftszonen oder durch die Stärkung der ländlichen Siedlungen? - Für (ökonomische) Potentialabschätzungen wesentlich ist im übrigen die Anbindung der Standorte an das übergeordnete Siedlungsnetz, das heißt ihre Erreichbarkeit. Daraus ergibt sich auch die Notwendigkeit der Differenzierung zwischen den Großräumen Mittelland, Voralpen, Jura und Alpen.

\section{Umbruch in der Landwirtschaft}

Ausgangspunkt der Diskussion um die Zukunft des ländlichen Raumes ist - zu Recht - der Umbruch in der Landwirtschaft; sie wird sukzessive zum Agrobusiness. Oder mit andern Worten: ökonomische Überlegungen werden zunehmend wichtiger, die öffentliche Hand verändert den Regulationsmodus. ${ }^{5}$ Dieser Umbruch ist aber - seinerseits - als Teil des gesamten Modernisierungsprozesses (Globalisierung, Regionalisierung) zu sehen. Sowohl aus ökonomischen wie auch aus ökologischen Gründen sind deshalb vor allem effizienzsteigernde Prozesse angesagt. Und der Rio-Nachfolgeprozeß verpflichtet auch die Schweiz, die Grundsätze der Nachhaltigkeit umzusetzen. Die knapper werdenden Ressourcen aller Art (Kapital, Boden, Umweltqualität, Lebensgrundlagen) verlangen eine Konzentration des Mitteleinsatzes, klare Prioritäten müssen gesetzt werden. Daneben besteht aber auch (zumindest in den demokratisch-föderalistisch verfaßten Staaten mit einer ökologisch-sozialen Marktwirtschaft) die gemeinsame Verpflichtung zum Ausgleich, welcher die Kohäsion fördern soll.

Ausgleichsprozesse sind allerdings nicht mehr denkbar als fester Anspruch, als automatisch zustehende Transferzahlungen, als wohlerworbene Rechte. Vielmehr basieren sie in jedem Fall auf Leistungsaufträgen, die zudem periodisch dem veränderten Umfeld angepaßt werden. Solche Leistungsaufträge sind Teil des neuen Gesellschaftsvertrages, um den heute gerungen wird.

Für die Landwirtschaft umschreibt BV 3 locties Abs. 2 klar, welcher Bereich solcherart privilegiert ist: bodenbewirtschaftende bäuerliche (Familien-)Betriebe können in Abweichung von der HGF gefördert werden. ${ }^{6} \mathrm{Im}$ übrigen ist der Markt zuständig für die Ressourcenzuteilung und Preisbildung. Neben den mannigfaltigen Transferzahlungen, welche nun - im Zuge der Agrarreform - auf «Mehr Markt, mehr Ökologie» getrimmt werden, ist auch die Landwirtschaftszone selbst nicht etwa als Schikane, sondern vielmehr als Privileg zu verstehen: Sie sichert durch die Spaltung des Bodenmarktes den bodenbewirtschaftenden Familienbetrieben (gemäß BV 3 locties) die nötige Produktionsgrundlage «Boden»

Martin Boesch, Prof. Dr., Universität St. Gallen, FWR-HSG/ Forschungsstelle für Wirtschaftsgeographie und Raumplanung 
zu deutlich günstigeren Kosten, als sie in einem unregulierten Markt anfallen würden. Dieser Privilegierung (in Abweichung von der HGF) fehlt die Verfassungsgrundlage, wenn sie gemäß dem Produktemodell auf bodenunabhängige, gebäude- und anlagengestützte Produktionsweisen ausgedehnt wird; dies gilt um so mehr für nichtlandwirtschaftliche (gewerbliche) Produkte. Umgekehrt wird so die fundamentale Bedeutung sichtbar, welche der mit dem RPG (1979) ${ }^{7}$ geschaffenen Landwirtschaftszone für die Bodenbewirtschaftung zukommt.

\section{Bodennutzung}

Es ist absehbar, daß sich die Bodennutzung in der Schweiz rasch weiter differenzieren wird: Außerhalb der Siedlungsgebiete wird es neben Gebieten mit intensiver landwirtschaftlicher Produktion vermehrt extensiv genutzte wie auch brachfallende, verwildernde Flächen geben. ${ }^{8}$ Keine Rhetorik wird diesen Prozeß stoppen, solange das aktuelle (globalisierte) ökonomische Umfeld besteht.

Diese Veränderung wird zumeist als Bedrohung verstanden. ${ }^{9}$ Und es ist in der Tat eine einschneidende, wohl auch schmerzliche Zäsur in der Entwicklung unserer Kulturlandschaft, eine eigentliche Wende: Nach der praktisch flächendeckenden Erschließung und landwirtschaftlichen Nutzung in der uns wohlvertrauten Form erfolgt nun umgekehrt ein selektiver Rückzug aus der Fläche. ${ }^{10}$

Man kann nun allerdings diese Entwicklung auch als Chance verstehen - als willkommenen Ansatz für die dringend nötige Ökologisierung: Sorgfältig geplante, konzeptionell gestützte Förderungsmaßnahmen mit gezieltem Einsatz der knappen Mittel führen zu einem Raummuster, welches

1. möglichst viel wertvolle Kulturlandschaft erhält und

2. ein ökologisches Optimum ermöglicht.

Dazu braucht es allerdings die entsprechenden ungestörten, unzerschnittenen naturnahen Räume; werden sie verstellt durch mannigfache landwirtschaftsfremde Nutzungen, fehlt das nötige ökologische Potential. ${ }^{11}$ Aus dem simplen «Mehr Ökologie» wird damit «Möglichst viel Ökologie für die verfügbaren Transfermittel» eine starke Herausforderung für die Agrarpolitik, ein klarer Leistungsauftrag der SteuerzahlerInnen und KonsumentInnen!

Als Trojanisches Pferd zeigt sich in diesem Zusammenhang der Begriff der Multifunktionalität der Landwirtschaft. Noch mit dem 7. Landwirtschaftsbericht (BLW 1992) bedeutete die Erkenntnis der Multifunktionalität einen erheblichen Fortschritt. Durch die Verankerung der vier Hauptaufgaben

\section{Nahrungsmittelversorgung;}

2. Pflege der natürlichen Lebensgrundlagen;

\section{Pflege der Kulturlandschaft und}

\section{4. dezentrale Besiedlung}

wurde der Leistungsauftrag der Landwirtschaft breit abgestützt. Folgerichtig nahm dann der neue Landwirtschaftsartikel BV 31 octies (1996) diese vier Hauptaufgaben unverändert auf. ${ }^{12}$

Die RPG-Revision $1998^{13}$ geht nun (in Art. 16 Abs. 1) darüber hinaus, indem neu auch vom «Erholungsraum» die Rede ist. Es ist wohl kaum anzunehmen, daß im 21. Jahrhundert unter «Erholungsraum» ein schlichtes Wanderparadies verstanden wird. Vielmehr ist davon auszugehen, daß die expandierende Freizeitgesellschaft bzw. die Freizeitindustrie laufend neue Bedürfnisse entwickelt; sie sind in aller Regel anlagengestützt und raumbeanspruchend, von der (motorisierten) Off-roadMobilität ganz zu schweigen. Und auf der Suche nach neuen einträglichen Nutzungsmöglichkeiten wird so die «Multifunktionalität» zum Freipaß für alle beliebigen Aktivitäten. Weit gefaßt - bis hin zu Freizeitparks, Sportanlagen, Disneyland, Survival Camps, Driving Ranges und andern ähnlichen Anlagen - wird hier Tür und Tor geöffnet für eine Umnutzung der offenen Flur. Sollen das Mittelland und Teile der Voralpen wirklich zu einem einzigen Erholungspark umgestaltet werden?

\section{Stärkung des ländlichen Raumes}

Mit der Frage nach der Stärkung des ländlichen Raumes kommen wir zum Kernpunkt der aktuellen Diskussion um die BAB-Verfahren. ${ }^{14}$ Das öffentliche Interesse an den ländlichen Gebieten ist klar und prioritär auf deren Stärkung als Ganzes ausgerichtet. ${ }^{15}$ Partikularinteressen wie die Entwicklungsmöglichkeiten einzelner Betriebe oder gar die Verwertung funktionsloser landwirtschaftlicher Gebäude haben sich (insbesondere bei Zielkonflikten) ein- und unterzuordnen. Was nicht der Stärkung des ländlichen Raumes als Ganzes dient oder sie gar in Frage stellt, soll nicht mehr weiter gefördert werden.

Was aber ist darunter zu verstehen? Das Konzept «Raumordnung Schweiz» enthält dazu klare Aussagen, ausgehend von den Entscheidungsprozessen ${ }^{16}$ in einer modernen, arbeitsteiligen Gesellschaft. Diese Aussagen zielen auf den Ausbau der bestehenden Siedlungen zu nachhaltig lebensfähigen Wohn- und Wirtschaftsstandorten, kompakt strukturiert, klar abgegrenzt gegenüber den möglichst großen Freiflächen, eingebunden ins übergeordnete Siedlungsnetz, angeschlossen an leistungsfähige Mobilitätssysteme im Umweltverbund. 
Diese Ziele sind nur erreichbar durch eine klare Absage an jede Verzettelung. Ein Blick auf die Investitionsvorhaben ${ }^{17}$ zeigt nämlich auf, daß diese räumliche Konzentration der relativ schwachen Impulse dringend nötig ist, damit der Abstand zwischen Stadt und Land nicht zu groß und die Attraktivität der Kleinzentren nicht allmählich zu gering wird. Verschiedene Studien wie auch die laufende Diskussion um Gemeindreformen (so in den Kantonen LU, SG, TI) zeigen zudem auf, daß die kritische Masse an Nachfragepotential für unterschiedliche Dienstleistungen, Institutionen und kommerzielle Angebote (Einzelhandel, ÖV, Gemeindeverwaltung, Service public) bei Siedlungseinheiten von 1000 bis gegen 2000 EinwohnerInnen liegt. Wer kaufmännisch rechnen muß, zieht sich heute schon aus der Fläche zurück. Die öffentliche Hand wird folgen müssen - je gezielter, dezidierter und früher desto besser. ${ }^{18}$ Es kommt nämlich dazu, daß Bau, Unterhalt und Benützung disperser Erschließungssysteme (wie Straßen, Ver- und Entsorgungssysteme, Informationsnetze) jährlich über 10 Milliarden Franken Zusatzkosten verursachen, verglichen mit kompakteren Siedlungsstrukturen (BOESCH/SCHMID 1998). Diese Mittel wird die öffentliche Hand, aber auch die Wirtschaft in Zukunft nicht mehr so leicht aufbringen können bzw. nutzbringender verwenden wollen (BSP 1997). Der Kanton Bern ist bereits daran, die Konsequenzen aus dieser Erkenntnis zu ziehen: Die Siedlungsstrukturen sollen viel dezidierter als bisher auf ein leistungsfähiges ÖV-Netz abgestimmt werden (RR-BE 1992).

Unter der wohl sehr realistischen Annahme knapp begrenzter Investitionsmittel (privat und öffentlich) für den ländlichen Raum folgt daraus klar, daß eine Strategie der Stärkung auf eine gezielte, geplante Fokussierung dieser Mittel hinauslaufen muß, nicht auf eine breite Verzettelung. Es ist niemandem gedient, wenn im ländlichen Raum überall verstreut etwas Weniges gemacht wird, was man langfristig nur als Fehlinvestition bezeichnen kann. Gerade das Gewerbe braucht dringend gut erreichbare Standorte und Synergieeffekte, um kostengünstig produzieren und auf den sich öffnenden Märkten bestehen zu können. Unter diesem Blickwinkel ist es volkswirtschaftlich nicht sinnvoll, einen nicht existenzfähigen Landwirtschaftsbetrieb (nach Art. 24b RPG) vor Ort um einen ebenso fragilen Gewerbeteil zu ergänzen; hingegen sind erfolgreiche und dynamische Unternehmen mit neuen Arbeitsplätzen in jedem ländlichen Kleinzentrum hoch willkommen. Es ist im übrigen auch nicht nachvollziehbar, und es entbehrt einer verfassungsmäßigen Grundlage, wenn Nebenerwerbslandwirte, die selbständig gewerblich tätig sind, derart privilegiert würden

1. gegenüber ihren Kollegen, die als Angestellte oft sehr weite Arbeitswege in Kauf nehmen müssen, oder

2. gegenüber ihren Konkurrenten, die von Gewerbezonen aus operieren.

\section{Die Aufgaben der Raumplanung}

Der Raumplanung kommt zur Umsetzung der skizzierten Strategie zur Stärkung des ländlichen Raumes ein hoher Stellenwert zu. Indem sie sich (u. a.) mit den langfristig wirksamen Investitionen in Bauten und Anlagen befaßt, unterstützt sie den Aufbau einer materiellen Struktur, auf der die angestrebten wirtschaftlichen und gesellschaftlichen Aktivitäten basieren können (LENDI/ ELSASSER 1991/175 ff.). Die heute geforderte Flexibilität, die Volatilität betrieblicher Bedürfnisse und Funktionen bzw. der entsprechenden unternehmerischen Entscheidungen kann für die Raumplanung als langfristig angelegte Aufgabe allerdings nicht das primäre Kriterium sein. Vielmehr gilt es, eine Rahmenordnung zu schaffen, die über die Tagesaktualität hinaus Bestand hat und auch allen möglichen zukünftigen Akteuren sinnvolle Optionen eröffnet, die nicht mit den öffentlichen Interessen kollidieren.

Dazu kommt (wie oben dargelegt), daß auch für die Allokation der landwirtschaftlichen Transferzahlungen klare raumplanerische Grundlagen nötig sind, um einen effizienten, gezielten Mitteleinsatz zu gewährleisten. $\mathrm{Zu}$ Recht sind die Entwicklungskonzepte II mit den Planungsinstrumenten eng gekoppelt; in diesen Koordinationsprozeß wird sich auch die Agrarpolitik eingliedern müssen.

Damit wird die ordnungspolitische Funktion der Raumplanung deutlich: Im Sinne eines Optimierungs- und Ausgleichsauftrages soll sie dort (und nur dort) das Wirken der Marktkräfte unterstützen, wo Förderung nötig ist, und es dort korrigieren, wo jene die öffentlichen Interessen tangieren. Dazu braucht es einen klar formulierten Gestaltungswillen. Ein bloßer Nachvollzug aller beliebigen Bedürfnisse hingegen macht Raumplanung (und damit auch die Raumplaner!) obsolet. ${ }^{19}$

\section{Beurteilung der Revisionsvorlage}

Gemessen an den ursprünglichen Intentionen ${ }^{20}$ ist im Laufe der parlamentarischen Debatte aus der Revision des RPG nur ein kleiner Schritt geworden, aber ein entscheidender Schritt in die falsche Richtung. Folgende Schwachstellen sind insbesondere zu verzeichnen:

- ungenügende Konsequenz bei der Umsetzung der Grundsätze einer «Nachhaltigen Raumentwicklung» sowie der «Raumordnung Schweiz» (BRP 1996, BRP 1998);

- ungenügende Kohärenz der Raumordnungspolitik mit der Reform der Agrarordnung (AP 2002);

- ungenügende Vollzugsfähigkeit der BAB-Verfahren.

Zusammenfassend ergibt sich, daß die Vorlage zur Revision des RPG ${ }^{21}$ überflüssig und kontraproduktiv ist. Überflüssig ist die Revision, weil die legitimen Bedürfnisse und Anliegen im Außenbereich auch ohne Revision des RPG realisiert werden können bzw. - wie ein 
Blick auf die Praxis zeigt - auch tatsächlich realisiert werden. Insbesondere steht einer Differenzierung der Landwirtschaftszone nichts im Wege, soweit es Bundesrecht betrifft; ${ }^{22}$ natürlich sind hier aber die Kantone gefordert! Und der Bund hätte schon bisher die Möglichkeit, auf eine allenfalls als nötig erachtete «Helvetisierung» der Art. 24-Bewilligungen hinzuwirken.

Damit stellt sich aber auch die Frage nach der Verhältnismäßigkeit des Revisionsprojektes: Aufwand und Ertrag stehen sich hier diametral gegenüber.

Kontraproduktiv ist die Vorlage, weil sie übertriebene Erwartungen in eine wirkliche Liberalisierung und Flexibilisierung der BAB-Verfahren weckt, diese dann aber so umfassend nicht eingelöst werden (können), es sei denn um den Preis allzu extensiver Auslegung. Die Frustration der Bauwilligen und der Baubehörden wird erheblich sein! Die Regelungsdichte und die Planungsbürokratie werden noch weiter ausgebaut. ${ }^{23}$ Dies hängt auch damit zusammen, daß mit der Revision eine ganze Reihe unbestimmter Rechtsbegriffe geschaffen wurde, die nun einen erheblichen Interpretationsbedarf (auf Stufe Exekutive wie auch Judikative) auslösen; das BRP hat bereits signalisiert, daß es in Zukunft seine «Aufsichtsfunktion» wohl signifikant verstärken müsse. ${ }^{24}$

Das Verständnis und die Bereitschaft zur Unterstützung der berechtigten Anliegen der Bauern - zu denken ist hier vor allem an die Leistungsaufträge für den ökologischen Ausgleich - wird in den urbanen Milieus weiter zurückgehen; der Rückhalt der Bauernschaft in der städtischen Bevölkerung schwindet angesichts immer neuer Forderungen nach weiteren Privilegien und Sondervorteilen. Vor dem Hintergrund der schwierigen Diskussion um Ausgleichsprozesse im Rahmen eines neuen Gesellschaftsvertrages sind solche Ungleichgewichte zu vermeiden.

Und letztlich werden die zentralen Anliegen der «Raumordnung Schweiz» (BRP 1996) unterlaufen, namentlich die Trennung Baugebiet/Nichtbaugebiet, die Verdichtung nach innen, die Verstärkung der Netzwerkidee, die Nachhaltigkeit der Raumstrukturen und der Mobilitätssysteme; und zwar in einem subtilen Dissipationsproze $\beta$, der schließlich die angestrebte Raumordnung auflöst und jede Raumplanung überflüssig machen könnte.

Die Umsetzung und Anwendung des Raumplanungsrechtes ist als Lernprozeß zu verstehen. Innovative Lösungen (z. B. im Kanton Graubünden) sind entwickelt worden, die zeigen, daß das RPG durch die Kantone sehr zweckmäßig umgesetzt werden kann. Man darf inzwischen durchaus von einer gewissen Konsolidierung der Rechtsanwendung sprechen. ${ }^{25}$ Die Revision des RPG in diesem heiklen Bereich der BAB-Verfahren würde diesen Lern- und Konsolidierungsprozeß unnötigerweise wieder zurückwerfen und zu neuen Unsicherheiten und Verzögerungen in den Verfahren führen. - Dazu kommt, daß das Problem der «unechten» Ausnahmen (Art. 24 RPG) auch durch die Revision nicht gelöst wird. ${ }^{26}$

\section{Zusammenfassung}

- Nachhaltige Entwicklung im ländlichen Raum umfaßt zwei primäre Gestaltungsaufträge: die Stärkung der Kleinzentren als Wohn- und Arbeitsstandorte sowie die Verbesserung des ökologischen Ausgleichs im Außenbereich.

- Beide Aufgaben bedingen eine Fokussierung der knappen Mittel (private Investitionen und öffentliche Transferzahlungen) nach klaren raumplanerischen Konzepten.

- Die Förderung der bodenbewirtschaftenden bäuerlichen Betriebe erfolgt durch den weiteren Ausbau der ökologischen Leistungsaufträge sowie deren Privilegierung in der für sie reservierten Landwirtschaftszone.

- Die Bodennutzung im Außenbereich wird sich rasch weiter differenzieren. Neben intensiv und extensiv genutztem Landwirtschaftsland werden (weiterhin) Flächen in erheblichem Ausmaße brachfallen. Dieses Potential zur Ökologisierung ist in die integrale Landschaftsplanung einzubeziehen. Ebenso ist in bezug auf die Bodenbewirtschaftung die Koordination zwischen Agrarpolitik und Raumordnung noch zu vertiefen.

- Für bodenunabhängige und landwirtschaftsfremde Produktionsanlagen sowie Freizeitanlagen und Ferienhäuser werden in Planungsverfahren geeignete Standorte festgelegt. Dabei ist der Außenbereich möglichst großflächig offenzuhalten, die Kleinzentren sind optimal zu stärken und die Anforderungen nachhaltiger Mobilitätssysteme zu berücksichtigen. In aller Regel werden also Flächen im bestehenden Baugebiet zugewiesen.

- Die RPG-Revision 1998 ist für den Vollzug dieser Aufgabe überflüssig und kontraproduktiv; es wäre ein Schritt in die falsche Richtung. Insbesondere würde eine Öffnung der Landwirtschaftszone sowohl die Stärkung der Kleinzentren wie auch die Ökologisierung des Außenbereiches erschweren.

- Statt dessen soll die Anwendung und Umsetzung des bestehenden Rechts in Zusammenarbeit zwischen Bund und Kantonen weiterentwickelt und konsolidiert werden.

\section{Anmerkungen}

Dieser Artikel ist eine überarbeitete Fassung des Referates "Nachhaltige Entwicklung im ländlichen Raum" anläßlich der VLP-Tagung vom 27.8.1998 zum Thema "Liberalisierung beim Bauen außerhalb der Bauzonen". Die Tagung thematisierte Pro und Kontra der anstehenden Revision des RPG 
(Lockerung der Bestimmungen zur Landwirtschaftszone sowie bei den Ausnahmen nach Art. 24). Vgl. dazu: Bundesgesetz über die Raumplanung (RPG), Änderung vom 20. März 1998. BBI 1998/1455. Zu dieser Debatte leistet auch der vorliegende Text einen kritischen Beitrag.

1 Vgl. dazu v.a. die Dokumente der Uno-Konferenz Habitat II (Istanbul 1996), das Europäische Raumentwicklungskonzept EUREK (Noordwjik 1997), die neue Regionalpolitik der EU (GD XVI) sowie die Arbeiten der ARL - Akademie für Raumforschung und Landesplanung, des BMBau/BBR Bundesamt für Bauwesen und Raumordnung und des IÖR Institut für Ökologische Raumentwicklung. - Ferner K.D. SCHNELL (1997); J. WOLF (1996).

2 V. HAUFF (1987).

${ }^{3}$ Bei der Nachführung der Bundesverfassung soll der Grundsatz explizit Erwähnung finden.

${ }^{4}$ Vgl. ebenso den Slogan "Preise statt Pläne" der Kommission "Stadt 2000" (BMBau). BBR 1998/IV.

${ }^{5}$ Die Bruttoerträge sind zwischen 1992 und 1997 um rund $30 \%$ gesunken, v.a. infolge der Reduktion der Produzentenrente (Abbau des Grenzschutzes). Bei den öffentlichen Transferzahlungen hat sich eine markante Verlagerung von den Subventionen zu den Direktzahlungen ergeben. Die eigentliche Wertschöpfung kann auf ca. 3 Mrd. CHF p.a. geschätzt werden.

${ }^{6}$ BV Art. 31octies Abs. 2: «Ergänzend zur zumutbaren Selbsthilfe der Landwirtschaft und nötigenfalls abweichend von der Handels- und Gewerbefreiheit fördert der Bund die bodenbewirtschaftenden bäuerlichen Betriebe." (Auszeichnung durch Verf.)

${ }^{7}$ Bundesgesetz über die Raumplanung (RPG). SR 700.

8 "Wildnis" kann sehr unterschiedliche, attraktive Ausprägungen zeigen. Vgl. z. B. die aktuelle Pro-Natura-Kampagne zur Revitalisierung der Gewässer ("Mehr Platz für Flüsse") und damit auch zur Aufwertung und Vergrößerung der Auengebiete (Pro Natura Spezial 4/98).

${ }^{9}$ W. BÄTZING W. (1997).

${ }^{10} \mathrm{Vgl}$. dazu auch die analoge Entwicklung in New England/ USA nach 1850.

11 Vgl. dazu die Strategieaussagen zum Bereich Natur- und Landschaftsraum in den "Grundzügen der Raumordnung Schweiz" (1996/37 ff.).

${ }^{12}$ BV Art. 31octies Abs. 1: "Der Bund sorgt dafür, daß die Landwirtschaft durch eine nachhaltige und auf den Markt ausgerichtete Produktion einen wesentlichen Beitrag leistet zur: a) sicheren Versorgung der Bevölkerung; b) Erhaltung der natürlichen Lebensgrundlagen und Pflege der Kulturlandschaft; c) dezentralen Besiedlung des Landes."

${ }^{13}$ Bundesgesetz über die Raumplanung (RPG), Änderung vom 20. März 1998. BBI 19981455.

${ }^{14}$ BAB - Bauen außerhalb der Bauzonen. Einerseits geht es um zonenkonformes Bauen in der Landwirtschaftszone, andererseits um Ausnahmen nach Art. 24 RPG.

$15 \mathrm{Vgl}$. die vier Strategiebereiche in den "Grundzügen der Raumordnung Schweiz" (1996/37):

1. Städtische Räume ordnen;

2. Ländliche Räume stärken;

3. Natur- und Landschaftsraum schonen;

4. Die Schweiz in Europa einbinden.

16 Gemeint sind hier die raumwirksamen Regulations- und Akkumulationsprozesse.

$17 \mathrm{Vgl}$. dazu die Statistik der Bauvorhaben (BFS $1987 \mathrm{ff}$.$) .$

$18 \mathrm{Vgl}$. z.B. die aktuelle Diskussion um Spitalstrukturen.

19 Vgl. dazu Mayer/Siebel (1998/10ff.) über das Dilemma einer Planung, welche sich ohne klare Zielorientierung pragmatisch aus allen Konflikten heraushalten möchte.
${ }^{20}$ Vgl. dazu die Motion Zimmerli (1991) sowie die Ergebnisse der Kommission Durrer (1994). Botschaft zu einer Teilrevision des Bundesgesetzes über die Raumplanung (RPG) vom 22. Mai 1996. BBI 1996/296ff.

21 Bundesgesetz über die Raumplanung (RPG), Änderung vom 20. März 1998. BBI 19981455.

22 Vgl. dazu die KPK-Studie "Lösungsansatz Planungsmodell». Solothurn 1995.

${ }^{23}$ Vgl. zum Beispiel die Art. 16a Abs. 3, 24b, 24c, 37a mit den entsprechenden Kommentaren der VLP.

${ }^{24} \mathrm{~F}$. Wegelin im "Bodenblatt" $1 / 98$

${ }^{25} \mathrm{Vgl}$. dazu etwa die abnehmende Zahl der Beschwerdefälle in BAB-Verfahren (Art. 24-Bewilligungen).

${ }^{26}$ Vgl. dazu schon K. VALLENDER (1986).

\section{Literatur}

ARL - AKADEMIE FÜR RAUMFORSCHUNG UND LANDESPLANUNG (Hrsg.) (1996): Nachhaltige Raumentwicklung. (= ARL - Arbeitsmaterial 234). Hannover 1996.

ARL - AKADEMIE FÜR RAUMFORSCHUNG UND LANDESPLANUNG (Hrsg.) (1997): Das Prinzip der nachhaltigen Entwicklung in der räumlichen Planung ( $=$ ARL-Arbeitsmaterial 238). Hannover 1997.

BÄTZING, W. (1997): Die Auflösung des ländlichen Raumes in der Postmoderne. In: Kommune 11/1997.

BBR - BUNDESAMT FÜR BAUWESEN UND RAUMORDNUNG (Hrsg.) (1998): Bausteine einer nachhaltigen Raumentwicklung (= BBR-Forschungen 88). Bonn 1998.

BFS - BUNDESAMT FÜR STATISTIK (1987ff.): Bau- und Wohnbaustatistik der Schweiz. Bauinvestitionen, Bauausgaben und -vorhaben, Wohnbautätigkeit. Bern $1987 \mathrm{ff}$.

BLW - BUNDESAMT FÜR LANDWIRTSCHAFT (Hrsg.) (1992): Siebter Landwirtschaftsbericht. Bern 1992.

BOESCH, M. / SCHMID, S. (1998): Mobilitätskosten und Siedlungsstruktur. Studie im Rahmen des NFP 41. Im Druck. BOESCH, M. (1996): Neue Perspektiven für den ländlichen Raum. In: BVR-Informationen (4) 20/33-44/1996.

BOESCH, M. (1997): Raumplanung in einer offenen Welt. In: BRP-Informationsheft 25 (3)/13-15/1997.

BRP - BUNDESAMT FÜR RAUMPLANUNG (Hrsg.) (1996): Grundzüge der Raumordnung Schweiz. Bern 1996.

BRP - BUNDESAMT FÜR RAUMPLANUNG (Hrsg.) (1998): Raumordnung und nachhaltige Entwicklung: Handlungsansätze für eine nachhaltige Raumentwicklung in der Schweiz. Bern 1998.

BSP - BUND SCHWEIZER PLANER (Hrsg.) (1997): Sparschäden bei den Infrastrukturen. Collage 1/97. Bern 1997. HAUFF, V. (1987): Unsere gemeinsame Zukunft. Der Brundtland-Bericht der Weltkommission für Umwelt und Entwicklung (Our Common Future). Greven 1987. 
IRMEN, E. / MEYER, H.v. (Hrsg.) (1996): Ländliche Räume. Ländliche Entwicklung im internationalen Vergleich (= Informationen zur Raumentwicklung 11/1996). Bonn 1996.

KPK - KANTONSPLANERKONFERENZ (Hrsg.) (1995): Lösungsansatz Planungsmodell. Räumliche Organisation der Landschaft. Leitvorstellungen als Grundlage für die Diskussion über eine Teilrevision des Raumplanungsrechtes in den Bereichen Landwirtschaft und Landschaft. Solothurn 1995. LENDI, M. / ELSASSER, H. (1991): Raumplanung in der Schweiz. Eine Einführung. Zürich 3\1991.

LINTZ, G. (1997): Grundlagen der Koordination von Umweltpolitik, regionaler Wirtschaftspolitik und Raumplanung (= IÖR-Schrift 22). Dresden 1997.

MAYER, H.-N. / SIEBEL, W. (1998): Neue Formen politischer Planung: IBA Emscher Park und Expo 2000 Hannover. In: DISP 134/4-11/1998.

REGIERUNGSRAT BE (1992): Bericht des Regierungsrates an den Großen Rat: Berner S-Bahn/Siedlung. Bern 1992. $\mathrm{ROCH}$, I. (Hrsg.) (1996): Umweltverträgliche regionale Entwicklung im Grenzraum und im ländlichen Raum. 4. IÖRSymposium 1995 (= IÖR-Schrift 19). Dresden 1996.

SCHNELL, K.D. (1997): Nachhaltige Raumordnung im Nichtsiedlungsgebiet. Zur Notwendigkeit einer kohärenten und aktiven Gestaltung des Außenraumes (= FWR-Publikationen 31). St. Gallen 1997.

SIEVERTS, TH. (1997): Zwischenstadt - Zwischen Ort und Welt, Raum und Zeit, Stadt und Land (= Bauwelt Fundamente 118). Braunschweig 1997.

VALLENDER, K. (1986): Ausnahmen von der Nutzungsordnung: Theoretische Grundlagen. In: SIV (Hrsg.) (1986): Rechtsfragen der Nutzungsordnung in der Raumplanung (= SIV-Veröffentlichungen NR 24). St. Gallen 1986. WOLF, J. (1996): Nachhaltige Raumentwicklung. Ein Beitrag zu einem neuen Leitbild der Raumordnung. Berlin 1996. 